\title{
The Method of Syntheses of Hardware and Software Complexes for Unmanned Aerial Vehicles Flight Control Systems Development
}

\author{
Andrey M. Ageev ${ }^{\mathbf{a}}$ and Ivan V. Makarov \\ ${ }^{a}$ Military Education and Research Centre of Military-Air Forces \\ «Military-Air Academy \\ Named After Professor N.E. Zhukovsky and Yu.A. Gagarin» \\ 54a Starykh Bolshevikov Str., Voronezh, 394064, Russia \\ ${ }^{b}$ «Autonomous Aerospace Systems - GeoService» \\ Research and Production Enterprise, LLC \\ 223 office, 3 Vuzovsky Side Str., Krasnoyarsk, 660025, Russia
}

Received 12.03.2016, received in revised form 27.06.2016, accepted 29.09.2016

\begin{abstract}
The method of design of hardware and software complexes for unmanned aerial vehicles flight control systems development, used by developers during the design and testing, is presents. The results of creation of a simulation model of short-range type unmanned aircraft flight dynamics, means of interfacing simulation models and flight control hardware and specialized software development, is considered. The recommendations on the use of the developed models and programs in the problems of the synthesis of unmanned aerial vehicles flight control systems are given.
\end{abstract}

Keywords: unmanned aerial vehicles, automatic flight control system, software and hardware complex, simulation model, simulation.

Citation: Ageev A.M., Makarov I.V. The method of syntheses of hardware and software complexes for unmanned aerial vehicles flight control systems development, J. Sib. Fed. Univ. Eng. technol., 2016, 9(8), 1267-1278. DOI: 10.17516/1999-494X2016-9-8-1267-1278.

(C) Siberian Federal University. All rights reserved

* Corresponding author E-mail address: ... 


\title{
Методика синтеза программно-аппаратных комплексов автоматизированной разработки систем управления полетом беспилотных летательных аппаратов
}

\author{
А.М. Агеев, И.В. Макаров \\ ${ }^{a}$ Военный учебно-научный цеентр Военно-воздушных сил \\ «Военно-воздушная академия \\ имени профессора Н.Е. Жуковского и Ю.А. Гагарина» \\ Россия, 394064, Воронеж, ул. Старых Большевиков, 54 а \\ ${ }^{6}$ ООО Научно-производственное предприятие \\ «Автономныле аэрокосмические системы «АВАКС-ГеоСервис» \\ Россия, 660025, Красноярск, пер. Вузовский, 3, 233
}

Предложена методика построения программно-аппаратных комплексов автоматизированной разработки систем управления полетом беспилотных летательных аппаратов, применяемых разработчиками на этапах проектирования и испытания. Рассмотрены результать создания имитационной модели динамики полета беспилотного летательного аппарата малой дальности самолетного типа, средств обеспечения информационного сопряжения имитационных моделей и аппаратных средств автоматизации управления, разработки соответствующего специиализированного программного обеспечения. Дань рекомендации по применению разработанных моделей и программ в задачах синтеза бортовых систем управления перспективных беспилотных летательных аппаратов.

Ключевые слова: беспилотный летательный аппарат, система автоматического управления полетом, программно-аппаратный комплекс, имитационная модель, имитационное моделирование.

\section{Введение}

В ближайшее десятилетие существенно увеличится количество комплексов с беспилотными летательными аппаратами (БЛА) при расширении ряда решаемых задач, что связано с увеличением высот, скоростей, дальности и продолжительности полета, точностных характеристик целевых нагрузок. Наметилась устойчивая тенденция автоматизации всех режимов полета БЛА, учитывая автоматический взлет/посадку, полет по заданному маршруту в районах патрулирования. Существующая практика разработки систем автоматического управления (САУ) БЛА основана на использовании классических схем на базе ПИД-регуляторов [1]. Расчет и настройка коэффициентов регуляторов САУ зачастую проводятся разработчиками эмпирически, не основываясь на аналитических расчетах, без привязки к конкретному планеру. Они требуют значительной коррекции на ранних стадиях испытаний и эксплуатации и в ряде случаев допускают неудовлетворительные летные характеристики и даже потери БЛА. На предприятиях промышленности, представленных многообразием фирм-производителей БЛА, отсутствует единый законченный научно обоснованный подход к разработке САУ полетом БЛА. Научно-исследовательские организации и конструкторские бюро заинтересованы в создании инструмента проведения научных исследований в области автоматизации управления БЛА с целью разработки новых алгоритмов управления, решения задач автоматизации 
взлета и посадки, других сложных задач управления и навигации. В то же время требования к низкой стоимости и малому времени на разработку беспилотных комплексов не позволяют закладывать в бюджет предприятий затраты на разработку или приобретение дорогостоящих специализированных программных продуктов, интеграцию их с пилотажно-навигационным оборудованием БЛА. Указанные обстоятельства определяют актуальность, практическую значимость и целесообразность выполнения исследований по обоснованию технологий для разработки недорогих, доступных программно-аппаратных комплексов как универсального инструмента для проведения научно-технических исследований и автоматизированной разработки систем управления полетом БЛА.

Основные задачи проведенного исследования:

1. Обоснование концепции и технологии построения программно-аппаратных комплексов исследования и автоматизированной разработки систем управления БЛА.

2. Разработка программных имитационных моделей динамики полета БЛА, элементов комплекса управления БЛА, земной поверхности, атмосферы.

3. Разработка аппаратуры обеспечения информационного сопряжения программных моделей БЛА и аппаратных средств автоматизации управления полетом БЛА.

4. Разработка специализированного управляющего программного обеспечения информационного сопряжения программных моделей БЛА и аппаратных средств автоматизации управления полетом БЛА.

5. Разработка рекомендаций по применению программно-аппаратного комплекса в задачах синтеза бортовых систем управления перспективных БЛА.

\section{Обоснование концепции}

\section{программно-аппаратных комплексов исследования}

и разработки систем автоматизации управления БЛА

При анализе состава и типовой структуры САУ БЛА, средств их исследования, разработки и испытания было обосновано, что на современном этапе программно-аппаратные комплексы исследования и автоматизированной разработки являются необходимым дополнением комплексов с БЛА, обеспечивающим всестороннюю поддержку изделия на всех стадиях его жизненного цикла [2]. Методологической основой таких комплексов может быть выбрана технология тестирования программного обеспечения САУ методом сравнения с эталонной моделью, применяемая при разработке систем управления пилотируемых и проектируемых в рамках опытно-конструкторских работ по созданию БЛА массой свыше 20 т, беспилотных ЛА [3]. С учетом существующего научно-технического задела была определена концепция и разработана технология создания программно-аппаратных комплексов исследования систем автоматизации управления ЛА, апробирована их работа на действующих макетах с использованием недорогих радиоуправляемых моделей [3]. Наработки в области имитационного моделирования, обеспечения аппаратного и информационного сопряжения программных моделей БЛА и аппаратных средств автоматизации управления полетом БЛА позволили обосновать тактикотехнические требования и приступить к разработке проекта научно-исследовательского программно-аппаратного комплекса на базе бортового комплекса управления (БКУ) беспилотного летательного аппарата [5]. Исследования были выполнены специалистами ВУНЦ ВВС 


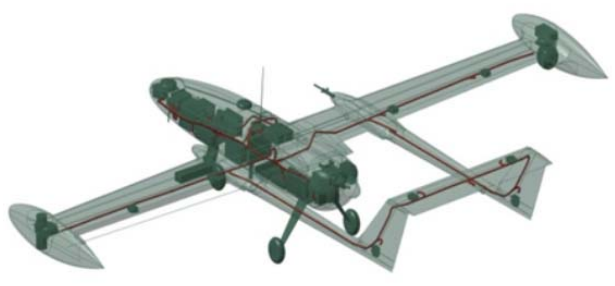

a

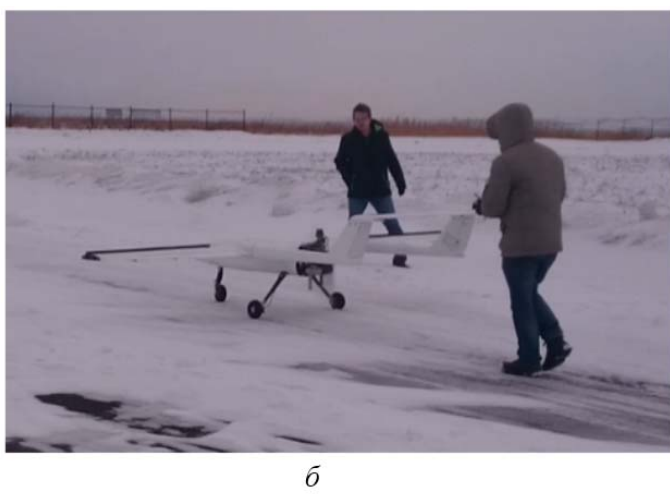

6

Рис. 1. Объект исследования: а - электронный макет БЛА; б - летные испытания БЛА

«ВВА» (г. Воронеж) и ООО НПП «Автономные аэрокосмические системы «АВАКС-ГеоСервис», (г. Красноярск) в рамках совместной опытно-конструкторской работы по созданию БЛА малой дальности «Гамма». На рис. $1 a$ представлен электронный макет БЛА. Внешний вид БЛА изображена на рис. 16 .

Основные технические решения, закладываемые в основу комплекса с БЛА: создание макроконфигурируемой системы автоматического управления в составе унифицированного БКУ БЛА, а также унифицированного устройства ввода-вывода для управления различными полезными нагрузками и дополнительным исследовательским оборудованием. Задача реконфигурируемости решается на всех уровнях за счет специализированного управляющего модульного программного обеспечения и централизации процессов управления всем комплексом в модуле управления движением на базе автопилота АП-05. На основании планируемого целевого назначения комплекса и совокупности решаемых задач были обоснованы требования и составлено техническое задание на комплект технических и программных средств разработчика и создания лабораторного стенда исследования системы управления полетом БЛА, разработаны структурная, функциональная и электрические схемы проектируемых систем. На рис. 2 представлена функциональная схема программного-аппаратного комплекса для выполнения имитационного моделирования управляющего программного обеспечения автопилота. Схема предполагает программно-аппаратное сопряжение бортового комплекса управления БЛА (автопилота) с программными средствами имитационного моделирования, визуализации и документирования результатов. Таким образом, образуется замкнутый программно-аппаратный цикл (hardware-in-the-loop), позволяющий проводить отладку функционирования алгоритмов управления САУ на этапах проектирования, испытания и модернизации БЛА.

\section{Разработка программных имитационных моделей беспилотного летательного аппарата}

При разработке программных имитационных моделей БЛА и их систем, земной поверхности, атмосферы основные усилия были направлены на соблюдение условий адекватности разрабатываемых моделей реальным объектам исследований. 


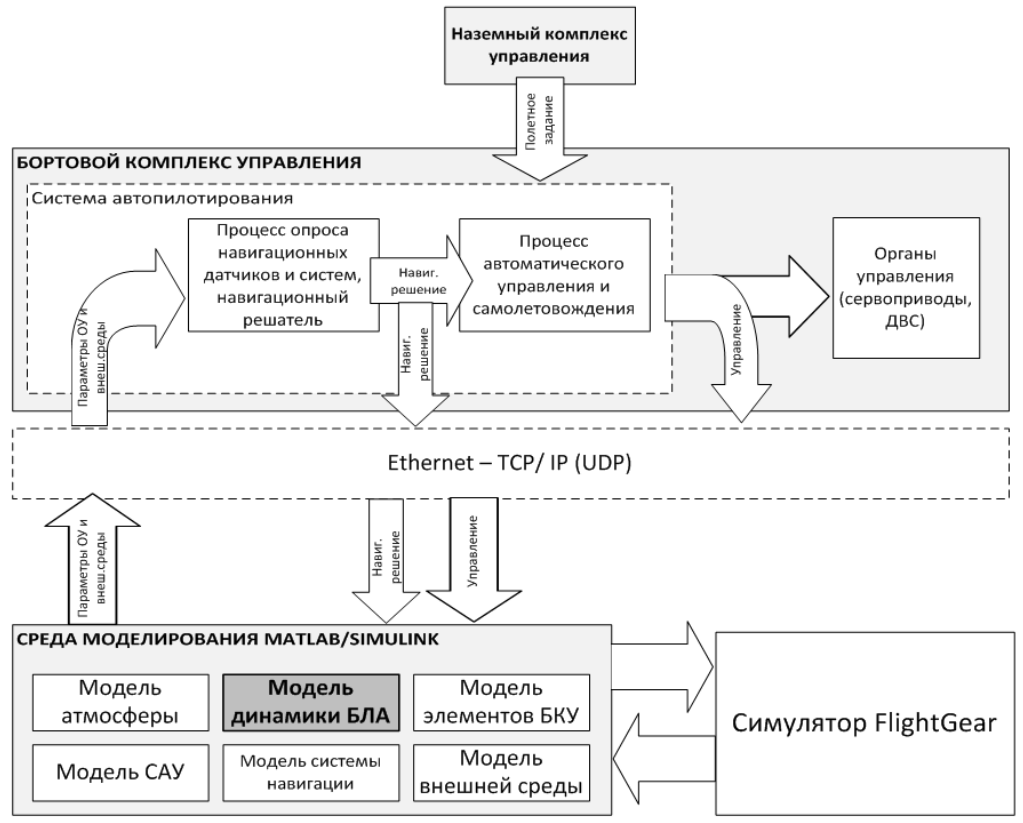

Рис. 2. Функциональная схема комплекса имитационного моделирования объекта управления для отработки программного обеспечения самолётовождения

Процесс создания модели был разделен на следующие основные этапы:

1) разработка трехмерных моделей планера БЛА и его элементов методами 3D-проектирования;

2) формирование банка данных аэродинамических характеристик БЛА на основе расчётов его трёхмерной модели в среде ANSYS CFX;

3) создание имитационной модели динамики полёта БЛА в MATLAB/Simulink;

4) создание имитационных моделей элементов комплекса управления БЛА в MATLAB/ Simulink;

5) комплекс контрольных расчетов и отработка функционирования модели;

6) создание средств визуализации и вывода результатов [6].

Разработка программных имитационных моделей динамики полета БЛА «Гамма», элементов комплекса управления БЛА, земной поверхности, атмосферы выполнена с использованием средств имитационного моделирования MATLAB/Simulink на основе аэродинамического расчета трехмерных моделей планера в комплексе аэрогидродинамических расчетов ANSYS CFX. Это позволило отказаться от дорогостоящих исследований с использованием продувок в аэродинамической трубе, однако было достаточным для создания адекватного банка аэродинамических данных рассматриваемого планера. Структура модели динамики БЛА (рис. 3) состоит из блоков вычисления сил и моментов, вычисления тяги двигателя, уравнений движения, моделей датчиков первичной информации, рулевых приводов, системы навигации и автоматического управления (СНАУ).

Модель динамики БЛА была дополнена нелинейными моделями элементов комплекса управления БЛА: силовой установки, датчиков первичной информации и рулевых приводов. 


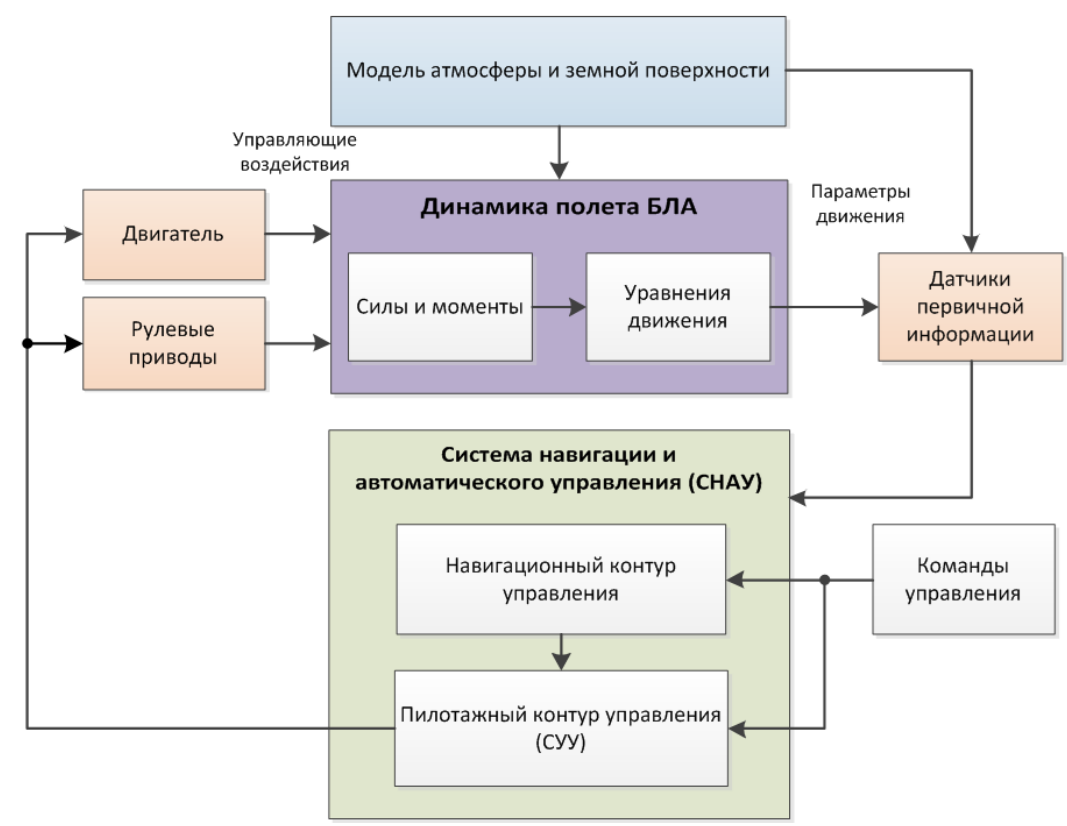

Рис. 3. Блок-схема полной модели динамики самолета

Математическая модель силовой установки основывается на статической характеристике двигателя и связывает положение дроссельной заслонки в процентах с тягой винтомоторной установки в ньютонах через частоту вращения воздушного винта. Также в модели считается часовой расход топлива. Математическая модель рулевого привода учитывает добротность привода, нелинейности типа «ограничение хода» привода, ограничение «скорости хода» привода, «зону нечувствительности», а также шарнирный момент, связанный с влиянием аэродинамических сил на рулевую поверхность в полете.

Результаты расчета модели представляются в виде графиков выходных параметров модели (скорость, высота, координаты, углы положения БЛА, угловые скорости и др.). После окончания моделирования имеется возможность выбрать интересующий участок полета и, используя широкие возможности средств Simulink по анализу графической информации (в том числе анализ переходных характеристик, частотный анализ и др.), провести исследование согласно поставленной задачи. Для визуализации результатов 3D-модель БЛА «Гамма» импортирована в виртуальную среду симулятора FlightGear, организована передача данных о пространственном перемещении, эволюциях, скорости полета и отклонениях рулей БЛА из модели в симулятор, а также передача данных о высоте над подстилающей поверхностью из симулятора в модель. Моделирование подстилающей поверхности было обеспечено путем подгрузки из

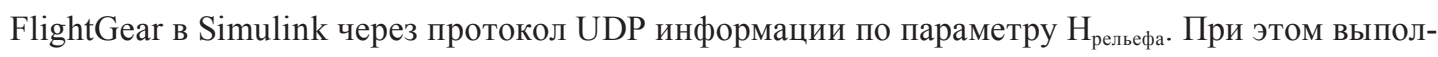
нялась предварительная привязка карты местности FlightGear к электронной карте местности системы Atlas путем указания начальных значений географических широты и долготы, а также начальной высоты и начального азимута.

С использованием полученной математической модели были рассчитаны балансировочные положения рулевых поверхностей, коэффициенты регуляторов пилотажного конту- 
ра БЛА, уточнены летно-технические характеристики аппарата, определены ограничения по высотам и скоростям полета. Также были определены потребные и располагаемые тяги силовой установки, максимально и минимально допустимые значения высот, скоростей полета и углов тангажа, произведен расчет взлета и посадки на ВПП. Расчеты проводились путем дачи тестовых входных воздействий, моделирования и последующей обработки его результатов, выполнения тестовых полетных заданий по отработке соответствующих режимов полета.

\section{Информационное сопряжение программных моделей БЛА и аппаратных средств автоматизации управления полетом БЛА}

При разработке аппаратуры обеспечения информационного сопряжения программных моделей БЛА и аппаратных средств автоматизации управления полетом БЛА был использован подход с централизованным управлением всем комплексом с помощью операционной системы реального времени QNX Neutrino, единым мультиплексным синхронным протоколом, связывающим все элементы комплекса. Операционная система QNX Neutrino работает в защищённом режиме, т. е. осуществляет виртуализацию адресов выполняемых процессов и отслеживает работу со сбойными указателями, что в значительной степени повышает надёжность всего программного комплекса [7].

В рамках решения задачи по автоматизации взлета, полета по заданному маршруту и посадки БЛА разработано специализированное управляющее программное обеспечение информационного сопряжения программных моделей БЛА и аппаратных средств автоматизации управления полетом БЛА $[8,9]$. Конфигурация модуля связи с внешними устройствами предусматривает одновременную работу механизма обмена на нескольких физических последовательных интерфейсах (RS485), при этом каждый физический интерфейс обслуживается одним потоком, который разграничивает по времени доступ внешних процессов к шине интерфейса, что гарантирует отсутствие коллизий. Модуль конфигурируется с внешнего файла в энергонезависимой памяти, где формулируется перечень интерфейсов, а также назначенные адреса устройств на интерфейсе и имена для доступа внешних процессов.

Специализированное программное обеспечение взаимодействия автопилота с моделями MATLAB/Simulink на первом этапе осуществляет чтение формата XML с приведением блоков редактора во внутренние программные узловые блоки САУ модуля пилотажного уровня f-cont, между которыми формируется связь в виде ссылок. Далее в процессе подготовки модели к итерированию осуществляется программная верификация модели, которая отслеживает уникальность имён сигналов и блоков, сверяет структуру САУ, проверяет результаты расчётов статических тестов.

Программно-аппаратное сопряжение математической имитационной Simulink-модели с автопилотом АП-05 реализовано путем передачи вектора информационного решения в конфигурационный XML-файл AП-05, а также передачи вектора управляющих сигналов обратно в модель по протоколу UDP с использованием специализированных модулей сетевого обмена MATLAB (рис. 4).

Данный способ позволяет при необходимости передавать и принимать и другие управляющие сигналы в автопилот, в том числе сигналы на управление различной целевой нагрузкой.

$$
-1273-
$$




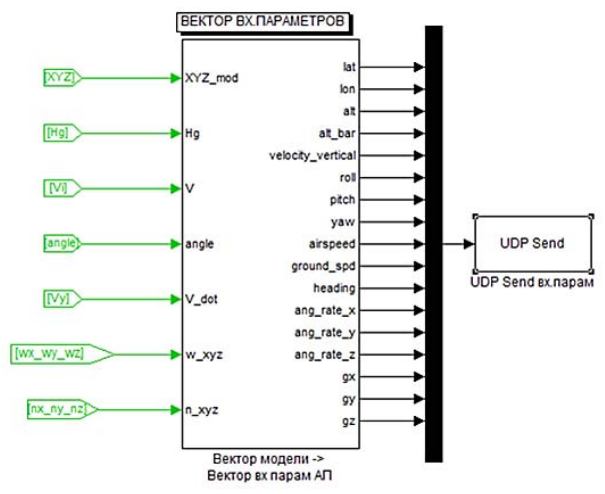

$a$

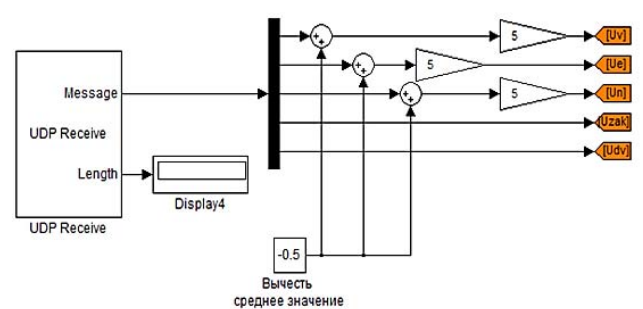

$\sigma$

Рис. 4. Simulink-схемы передачи и приема команд между моделью и автопилотом: а - передача вектора информационного решения модели в автопилот; б - передача вектора управления автопилота в вектор управления модели

\section{Рекомендации по применению программно-аппаратного комплекса в задачах анализа и синтеза бортовых систем управления БЛА}

При разработке рекомендаций по применению программно-аппаратного комплекса в задачах синтеза бортовых систем управления перспективных БЛА основной акцент сделан на прикладные аспекты исследования систем автоматического управления, синтеза алгоритмов их управления. В работе рассмотрена методика расчета характеристик системы управления БЛА с применением разработанного комплекса моделей на примере расчета коэффициентов регуляторов продольного канала стабилизации управления полетом БЛА в задачах автоматизации взлета, полета по маршруту и посадки БЛА на ВПП. Методика основана на синтезе контуров ПИД-регуляторов для разработанной модели БЛА с применением инструментария Control and Estimation Tools Manager, включающего Simulink Control Design и PID Tuner.

Методика расчета характеристик системы управления БЛА с применением разработанного комплекса моделей рассмотрена на примере расчета коэффициентов регуляторов продольного канала стабилизации управления полетом БЛА.

Задачу расчета продольного канала управления можно декомпозировать на следующие подзадачи (этапы):

1. Предъявление требований к характеристикам устойчивости и управляемости в выделенном канале движения.

2. Составление структурной схемы динамики полета БЛА с системой управления.

3. Декомпозиция полной математической модели динамики полета БЛА на изолированные движения и выделение необходимого канала путем исключения составляющих, оказывающих несущественное влияние на динамику исследуемого канала.

4. Линеаризация выделенного канала известными методами [10].

5. Составление законов управления выделенным каналом.

6. Разработка структурной схемы управления выделенным каналом.

7. Составление имитационной Simulink-модели канала управления.

8. Расчет коэффициентов регуляторов известными способами [11]. 
9. Моделирование работы системы «БЛА-САУ» на упрощенной модели с выделенным каналом.

10. Моделирование работы системы «БЛА-САУ» на полной модели БЛА.

11. Повторение этапов 4-10 до достижения требуемого результата (требуемых характеристик устойчивости и управляемости).

Поясним суть решения указанных задач на примере расчета контура стабилизации продольного канала управления БЛА. Расчет целесообразно начинать на модели, для которой проведен расчет балансировочных значений положения рулей и дроссельной заслонки двигателя для установившихся режимов полета. Первый этап подготовительный и предъявляет требования к качеству управления БЛА в продольном канале (время регулирования, перерегулирование и др.). На втором этапе собиралась простейшая система стабилизации БЛА в продольном канале, представленная каскадом ПИД-регуляторов (PID_wz, PID_tang, PID_H и PID_V). На третьем этапе выделялся изолированный канал управления путем назначения входов и выходов линеаризуемой системы. В нашем случае входами линеаризуемой системы были выходы параметров состояния модели в продольном движении (угол тангажа, угловая скорость тангажа, высота, скорость, нормальная перегрузка). В качестве выходов линеаризуемой системы выступали входы вектора управления по каналам руля высоты и положения дроссельной заслонки. Четвертый этап - процесс линеаризации - реализовывался с помощью утилиты Control and Estimation Tools Manager. Результаты линеаризации могут быть представлены в виде системы в пространстве состояния либо в виде передаточных функций от каждого входа по каждому выходу системы. Этапы 5-7: составление законов управления, структурных схем и моделей управления выделенным каналом, для чего в MATLAB были coбраны схемы трех аналогичных ПИД-регуляторов, управляющих креном тангажом и рысканием. На входы регуляторов приходят значения текущего угла (крена, рыскания) или высоты полета и требуемого желаемого значения (задатчика). Этап 8 - расчет регуляторов замкнутой системы одним из существующих способов расчета коэффициентов ПИД-регуляторов, например метод Циглера-Николса [12], метод биноминального распределения корней (метод Ньютона) [13] и др. В Matlab/Simulink для этой цели применяется инструментарий Simulink Control Design и, в частности, инструмент настройки регуляторов PID Tuner. На этапах 9-11 происходит моделирование работы системы «БЛА-САУ» на упрощенной модели с рассчитанными коэффициентами регуляторов, проверка соответствия параметров переходных процессов (по тангажу) заданным, моделирование работы системы «БЛА-САУ» с данными значениями регуляторов на полной модели БЛА. В случае несоответствия характеристик требуемым возвращаемся к этапу расчета регуляторов (этап 8) либо выбираем иную схему стабилизации (этап 2) и повторяем весь процесс.

\section{Результаты имитационного моделирования}

Применительно к задаче наземной отработки взлета и посадки было проведено имитационное моделирование этапов полета с использованием разработанных моделей. Результаты моделирования подтвердили, что имитационный комплекс позволяет достоверно моделировать изменение пилотажных и навигационных параметров в процессе выполнения алгоритмов взлёта и посадки. Заложена возможность учёта ветровых нагрузок с произвольной скоростью 


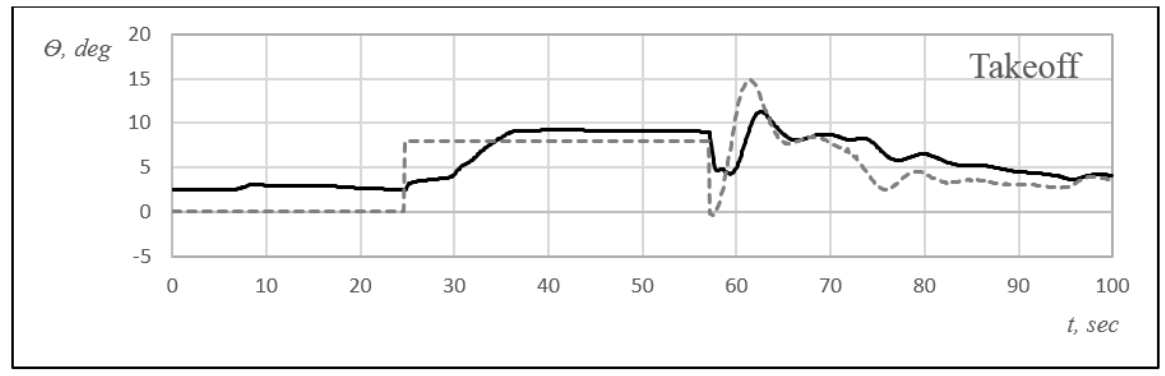

$a$

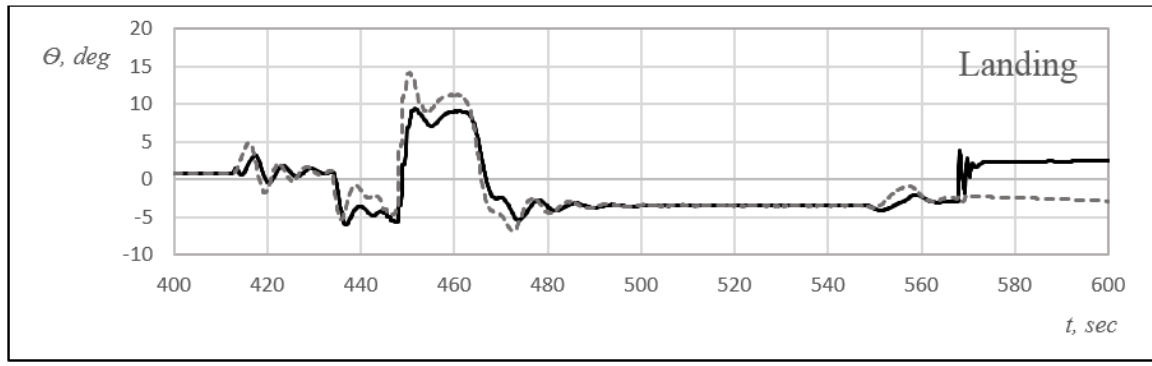

6

Рис. 5. Графики угла тангажа и входа регулятора тангажа при моделировании взлёта (а) и посадки (б) БЛА (сплошная линия - угол тангажа, пунктирная - вход регулятора тангажа)

и направлением. На рис. 5 представлены графики, иллюстрирующие пример моделирования этапов взлета и посадки БЛА в продольном движении.

\section{Заключение}

Отработанные при создании комплекса методики позволят обеспечить замкнутый цикл исследования и разработки систем управления БЛА, минимизировать финансовые и временные затраты на разработку программного обеспечения САУ БЛА до начала процесса лётных испытаний. Результаты могут быть использованы при проектировании бортовых систем управления БЛА самолетного типа малой и средней дальности, позволят решать разнообразные инженерные и исследовательские задачи на этапах их испытаний и модернизации. Подобные комплексы также дадут возможность решать задачи по оценке эффективности функционирования и применения комплексов с БЛА (в части, касающейся систем управления и навигации) на ранних стадиях разработки и оценки технических проектов. Полученные в ходе выполнения работы результаты являются основой для разработки научно-исследовательской базы исследования систем автоматизации управления БЛА.

Работа выполнена при поддержке гранта РФФИ № 15-08-02611А.

\section{Список литературы}

[1] Valavanis K.P., Vachtsevanos G.J. Handbook of Unmanned Aerial Vehicles. New York+London, Springer Science+Business Media Dordrecht, 2015. 526-710. 
[2] Моисеев В.С. Прикладная теория управления беспилотными летательными аппаратами. Казань: РЦМКО, 2013. 768 с. [Moiseev V.S. Applied theory of unmanned aircraft control. Kazan: RCMCO, 2013, 768 p (in Russian)].

[3] Сильвестров М.М., Бегичев Ю.И., Варочко А.Г. и др. Технология создания комплексов управления многочелевых беспилотных и пилотируемых аэрокосмических комплексов М.: Издательство МБА, 2014. 296 с. [Silvestrov M.M., Begichev Y.I., Varochko A.G. Technology of multipurpose manned and unmanned aerospace complexes control systems development. Moscow: MBA, 2014, 296 p (in Russian)].

[4] Агеев А. М., Чепурной Д.Ю, Садырев Е.А. Разработка программно-аппаратных средств исследования систем автоматизации управления полетом летательных аппаратов, Сборник статей по материалам докладов ХХІV Межвузовской НПК «Перспектива-2014». Авиаиионное оборудование, Воронеж: ВУНЦ ВВС, 2014, 73-78. [Ageev A.M., Chepurnoy D.Y., Sadirev E.A. XXIV Interuniversity conference «Perspective 2014». Aeronautical equipment, Voronezh, Air Force Academy, 2014, 73-78 p (in Russian)].

[5] Агеев А.М., Попов А.С. Работы по созданию универсальной научно-исследовательской платформы на базе БПЛА. Сборник трудов Всероссийской научно-технической конференции «ХІ Научные чтения по авиации, посвященные памяти Н. Е. Жуковского» (г. Москва 17-18 апреля 2014 г.), М: ВВИА, 2014. 123-125. [Ageev A.M., Popov A.S. All-Russian Scientific and Technical Conference «XI Scientific Readings on aviation memory of N.E. Zhukovsky» (Moscow, April 17-18, 2014), M: VVIA, 2014. 123-125. (in Russian)].

[6] Агеев А.М., Ищук И.Н., Попов А.С. Методика синтеза метеозависимой имитационной модели динамики полёта беспилотного летательного аппарата по данным расчета в ANSYS CFX. Вестник Воронежского государственного университета. Серия: Системный анализ и информационные технологии, 2015, 2. [Ageev A.M., Ishuk I.N., Popov A.S. Methods of synthesis meteodependent flight dynamics simulation model of an unmanned aerial vehicle according to the calculation in ANSYS CFX. Bulletin of Voronezh State University. Series: System Analysis and Information Technology, 2015, 2. (in Russian)].

[7] Makarov I. Control and Communications (SIBCON), International Siberian Conference IEEE, 2011. p. 152-154.

[8] Макаров И.В., Хорошко А.Ю. Создание конфигурируемой системы автоматического управления в составе унифицированного комплекса управления беспилотными летательными аппаратами. [Makarov I.V., Horoshko A.Y. Creation of configurable automatic control system in the composition of unification UAV flight control system. URL: http://www.uav-siberia.com/content/ sozdanie-configuriruemoj-sistemy-avtomaticheskogo-upravleniya-v-sostave-unificirovannogo (in Russian)].

[9] Макаров И.В. Имитационное моделирование полёта комплекса беспилотного летательного аппарата. Вестник СибГАУ. 6 (32), 2010, 78-82. [Makarov I.V. Simulation modeling of unmanned aerial vehicle complex flight// Bulletin Siberian State Aerospace University. 6 (32), 2010, 78-82 (in Russian)].

[10] Буков В.Н. Адаптивные прогнозирующие системы управления полетом. М.: Наука, 1987. 232 c. [Bukov V.N. Adaptive predictive flight control system. Moscow: Nauka, 1987. 232 p. (in Russian)]. 
[11] А.А. Красовский. Справочник по теории автоматического управления. М.: Nauka, 1987. [Handbook of automatic control theory. Ed. A.A. Krasovsky. Moscow: Science, 1987 (in Russian)].

[12] Ziegler J.G., Nichols N.B. Optimum settings for automatic controllers. Trans. ASME, 64, 759-768.

[13] Chien K.L., Hrones J.A., Reswick J.B. On automatic control of generalized passive systems. Trans. ASME, 74, 175-185. 